\title{
A continuum damage model to simulate failure in composite plates under uniaxial compression
}

\author{
D. Tumino*, F. Cappello, G. Catalanotti \\ Dipartimento di Meccanica, Universita' degli Studi di Palermo, V.le delle Scienze - 90128 Palermo, Italy
}

Received 17 October 2006; accepted in revised form 9 December 2006

\begin{abstract}
Experimental investigations and numerical simulations are performed in order to numerically predict the buckling behaviour of thin composite laminated specimens. Experiments are aimed at two objectives: the first is to completely characterize the carbon/epoxy material under simple loading configurations, the second is to test this material in buckling and post-buckling situations. The data collected with the first campaign of experiments are used to obtain the strength parameters required to define a damage model based on the failure theory by Tsai-Wu. This model is implemented in a Finite Element (FE) code and numerical simulations of buckling are executed for unidirectional and cross-ply laminates; results are in good agreement with experiments both in terms of determination of the critical loads and prediction of failure during post-buckling.
\end{abstract}

Keywords: polymer composites, mechanical properties, modelling and simulation

\section{Introduction}

The diffusion of composite materials in several fields of engineering and their application to important aspects of human lives are only few reasons for the relevance of the complete comprehension of their behaviour. Differently from conventional materials, composites are subjected to particular damaging processes that lower their capabilities up to their final failure. Carbon Fibre Reinforced Plastics (CFRP) are widely used for hi-tech applications in mechanics, aeronautics and astronautics especially because, compared to conventional materials, they offer a higher specific modulus of elasticity. They are often used as thin plates or as slender beams.

When dealing with thin components, problems arise if compressive conditions are present, because small values of loads can cause instability and then failure of the material at lower load levels than those predicted by compressive strength. Different consequences of such phenomenon can be found: matrix failure, fibres failure, interlaminar delamination.

For beam-shaped isotropic structures these critical loads can be evaluated with the Euler's theory [1] or with more sophisticated shear-deformation theories [2,3]. Analytical results carried out for laminated composites, are often affected by an overestimation of the critical loads [4]. Factors like orthotropy, asymmetry in traction-compression, edge effects or local damaging can provoke great differences between buckling tests and analytical theories.

Improvements are offered by numerical FE simulations [5-7] by which laminated composites can be reproduced with their intrinsic orthotropic behaviour. With FEM two different approaches can be adopted to solve buckling problems: linear and non-linear ones [8]. Linear buckling furnishes information when the structure has reached the neutral equilibrium between external loads and

*Corresponding author, e-mail: tumino@dima.unipa.it

(C) BME-PT and GTE 
elastic reactions (bifurcation point), under the assumptions that the load-displacements diagram is linear as load increases and large displacements occur just at the buckling moment. Non-linear buckling with FEM allows one to consider the presence of large deflections and damaging of material; this means that not only the bifurcation point but also pre- and post-buckling phases can be studied without any restrictive hypothesis. A previous work by the authors [8] demonstrates the influence of orthotropy, edge effect and stacking sequence of the plies on the lowering of the critical load predicted by analytical theories.

In this work a continuum damage model is proposed, based on the failure criterion by Tsai-Wu, to reproduce local damaging of composites. This model, together with an unsymmetric traction-compression constitutive model, is applied to the simulation of buckling and post-buckling of CFRP specimens. Results obtained by characterization tests give the parameters required to completely define the model. This model is used to study the buckling and post-buckling behaviour of composites. Results obtained by FEM simulations are compared with experimental tests on unidirectional and cross-ply laminates.

\section{Method}

Several failure criteria are available for composite materials, based on principles like the maximum stress, maximum strain or maximum energy for deformation [9]. Simple criteria like the maximum stress (or strain) state that failure occurs when the stress (or the strain) in one of the principal directions of the material reaches a limit value (assumed as the same in traction and compression). A more sophisticated criteria is the one by Tsai-Hill where failure is supposed to occur when an energy-related expression is satisfied; with this criterion the interaction between the various components of stress is accounted for.

Tsai-Wu criterion can be expressed in terms of stress or strain [10]; in this work the second formulation is used, where the failure condition can be written as:

$$
\begin{gathered}
\Psi=G_{11} \varepsilon_{1}^{2}+2 G_{12} \varepsilon_{1} \varepsilon_{2}+G_{22} \varepsilon_{2}^{2}+ \\
G_{66} \gamma_{12}^{2}+G_{1} \varepsilon_{1}+G_{2} \varepsilon_{2}=1
\end{gathered}
$$

where $\Psi$ is the Tsai-Wu index, $G_{i j}$ and $G_{i}$ are known as strength parameters and are characteristic of the material; they can be related to experimental data from six types of test by measuring the level of strain at failure in cases of: traction and compression in longitudinal direction (direction 1), traction and compression in transversal direction (direction 2), pure shear and biaxial state. The following relations are used to estimate these parameters:

$$
\begin{aligned}
& G_{11}=-\frac{1}{\varepsilon_{1 u T} \varepsilon_{1 u C}} ; G_{1}=\frac{1}{\varepsilon_{1 u T}}+\frac{1}{\varepsilon_{1 u C}} \\
& G_{22}=-\frac{1}{\varepsilon_{2 u T} \varepsilon_{2 u C}} ; G_{2}=\frac{1}{\varepsilon_{2 u T}}+\frac{1}{\varepsilon_{2 u C}} \\
& G_{66}=\frac{1}{\gamma_{12 u}^{2}}
\end{aligned}
$$

where $\varepsilon_{i u T}$ (for $i=1,2$ ) is the strain at failure in uniaxial traction, $\varepsilon_{i u C}$ (for $i=1,2$ ) is the strain at failure in uniaxial compression, $\gamma_{12 u}$ is the strain at failure in pure shear.

The term $G_{12}$ must be obtained from biaxial tests, but is usually taken as either of the following two values:

$$
G_{12}=0 \text { or } G_{12}=-0.5 \sqrt{G_{11} G_{22}}
$$

\subsection{Damage Mechanics}

We consider a volume of material free of damage if no cracks or cavities can be observed at the microscopic scale. The opposite state is the fracture of the volume element. The theory of damage describes the phenomena between the virgin state of the material and the macroscopic onset of crack $[11,12]$. The volume element must be of sufficiently large size compared to the inhomogeneities of the composite material. In Figure 1 this volume

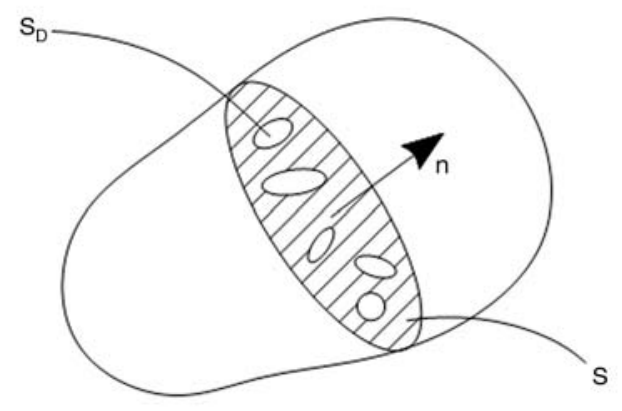

Figure 1. Representative volume element for damage mechanics 
is depicted. One section of this element is related to its normal and to his area $S$. Due to the presence of defects, an effective area for resistance can be found. The total area of defects, therefore, is:

$S_{D}=S-\tilde{S}$

The local damage related to the direction $\mathbf{n}$ is defined as:

$D_{n}=\frac{S_{D}}{S}$

Under the hypothesis of isotropic damage, the dependence on the normal $\mathbf{n}$ can be neglected, i.e.:

$$
D=D_{n} \forall \mathbf{n}
$$

The damage $D$ is a scalar assuming values between 0 and 1 . For $D=0$ the material is undamaged, for $0<D<1$ the material is damaged, for $D=1$ complete failure occurs.

The definition of damage leads to the introduction of the effective stress $\tilde{\sigma}$ :

$\widetilde{\sigma}=\frac{\sigma S}{\tilde{S}}=\frac{\sigma}{(1-D)}$

where $\sigma$ is the nominal stress.

Assuming that the strain is affected only by the effective stress [11], we can write for uniaxial stress state:

$\varepsilon=\frac{\tilde{\sigma}}{E}=\frac{\sigma}{(1-D) E}$

Rewriting Eq. (10) as:

$\sigma=E(1-D) \varepsilon=\tilde{E} \varepsilon$

we can treat $\tilde{E}$ as the elastic modulus of the damaged material.

The quantitative evaluation of damage is not a trivial issue, it must be linked to a variable that is able to characterize the phenomenon. Several papers can be found in literature where the constitutive equations of the materials is function of a scalar variable of damage. Most of these works [11-14] assume that the damage is related to an equivalent expression of the strain and to the load-history of the material. In [15] a damage loading function $f$ is defined as a threshold domain that the equivalent strain has to cross in order to generate damage in the material. In the works above mentioned, isotropic damage is considered. An orthotropic formulation of the damage can be found in [16] where different aspects of composite damaging are studied, i.e. debonding, matrix and fibre cracking.

In the present work we propose to link the damage $D$ not directly to an equivalent expression of the strain but to the Tsai-Wu index $\Psi$, that, as mentioned before, is an entity representing the multiaxial strain state in a composite material and is tightly related to the experimental values of strength at failure. The crucial point is how to relate the damage $D$ to the index $\Psi$. To do that, a simple power law is adopted:

$D=\Psi^{\alpha}$

where the parameter $\alpha$ must be tuned with experimental data obtained from characterization tests. Due to the definition of $\Psi$ given in Eq. (1), Eq. (12) can be interpreted as a strain-based expression of the damage.

Because of the brittle behaviour characteristic of composite materials, damaging process should be represented in a way that once damage starts to grow then failure occurs very rapidly. Let us consider, for example, the case of uniaxial traction in direction 1, with the simplified assumption that $\varepsilon_{1 u C}=-\varepsilon_{1 u T}$. Replacing Eq. (2a) and (2b) in Eq. (1), we have:

$\Psi=\left(\frac{\varepsilon_{1}}{\varepsilon_{1 u T}}\right)^{2}$

Then, dividing both terms of Eq. (11) by $\varepsilon_{1 u T}$ and substituting Eq. (12), we can write:

$\frac{\sigma_{1}}{E_{11} \varepsilon_{1 u T}}=(1-D) \frac{\varepsilon_{1}}{\varepsilon_{1 u T}}=\left[1-\left(\frac{\varepsilon_{1}}{\varepsilon_{1 u T}}\right)^{2 \alpha}\right] \frac{\varepsilon_{1}}{\varepsilon_{1 u T}}$

In Figure 2, Eq. (14) is plotted for different values of $\alpha$. The plot clearly explains how for very high values of $\alpha$ the damage model based on the Tsai$\mathrm{Wu}$ index approaches to the maximum stress (or strain) failure criterion (MSC). An optimal value for $\alpha$ must be chosen considering two different aspects: high values well represent the brittle failure mechanism of composites, small values simplify the computational procedure. Furthermore, in order to satisfy the condition of isotropy, $\alpha$ must be unique and independent from the direction of the material. 


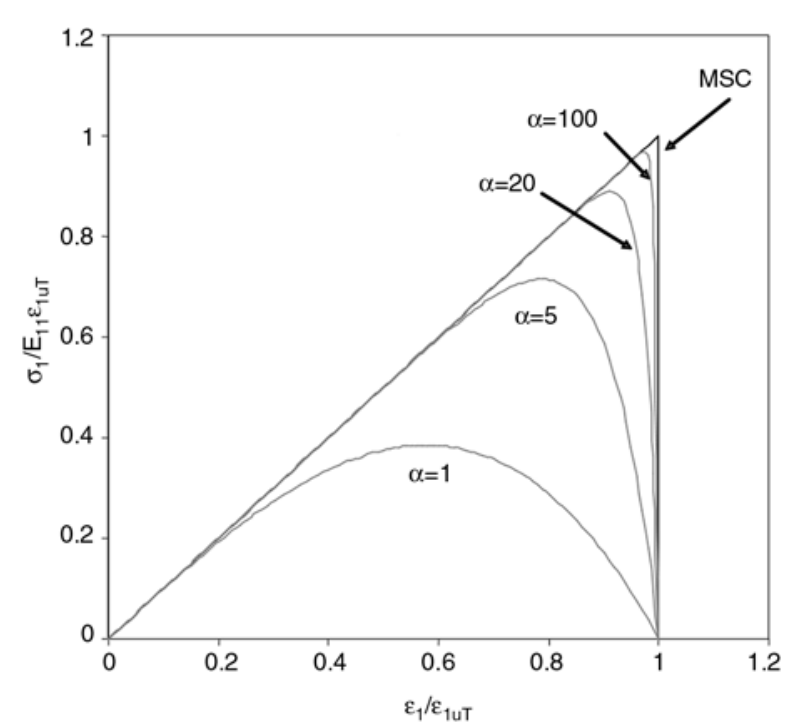

Figure 2. Constitutive model with damage for different values for $\alpha$

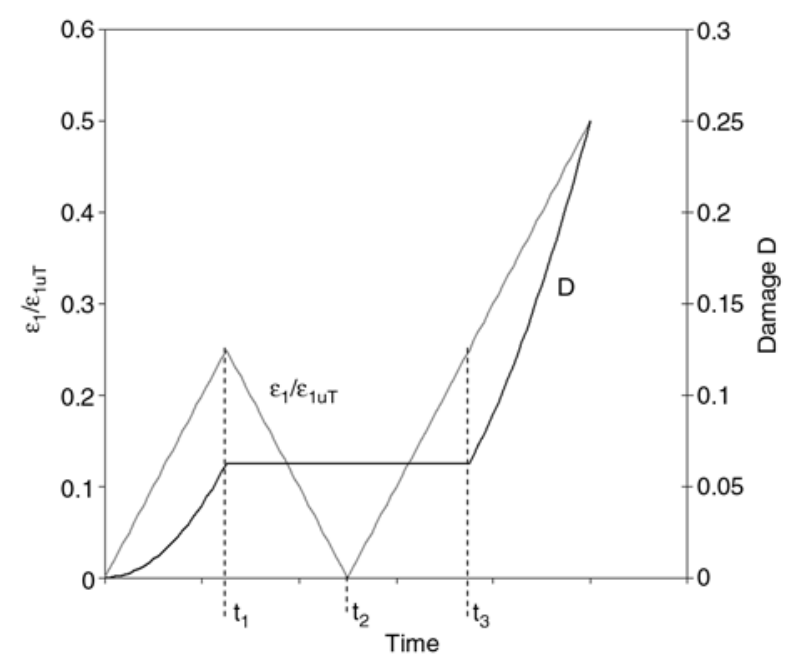

Figure 3. Deformation and related damage history with time $(\alpha=1)$

Another important characteristic of this damage formulation is its irreversibility: $D$ must grow monotonically from 0 to 1 . In Figure 3 is reported an example of the damage history related to the strain history with time: $D$ increases with $\varepsilon_{1} / \varepsilon_{1 u T}$ (time $\left.0-t_{1}\right)$, then as $\varepsilon_{1} / \varepsilon_{1 u T}$ decreases $D$ is constant $\left(t_{1}-t_{2}\right)$, also when $\varepsilon_{1} / \varepsilon_{1 u T}$ restarts growing $D$ is constant $\left(t_{2}-t_{3}\right)$; in the last interval $\left(t_{3}-t_{4}\right), D$ and $\varepsilon_{1} / \varepsilon_{1 u T}$ increase.

\section{Results}

The material used for the test is a carbon-epoxy composite, obtained laminating the dry patches of carbon fibre by PROCHIMA s.n.c., with an epoxy resin by MATES Italiana s.r.l.. The unidirectional 12-laminae plate so obtained is cured at room-temperature for 12 hours and then at $60^{\circ} \mathrm{C}$ for 12 hours. The specimens are machined from the original plate and instrumented with strain gauges. A crossply laminate is also manufactured with a $[0 / 90]_{3 s}$ lay-up. A small difference in the fibre volume fraction is observed: $V_{f}=50 \%$ for the unidirectional laminate, while $V_{f}=42 \%$ for the cross-ply laminate.

\subsection{Characterization tests}

Elastic characteristics of the material are found by testing specimens whose geometric dimensions are indicated by the ASTM standards [17-19]. In Table 1, these characteristics are summarized for the unidirectional material. A slight difference between the modulii in traction and compression can be noticed. This can be due to the crimped aspect of the fibres and is the reason for the need of an unsymmetric traction-compression constitutive model for the FEM analyses. To obtain the strength parameters defined in Eq. $(2,3,4)$, five test are performed on the material $\left(G_{12}\right.$ is estimated via Eq. (5b) because of the great difficulty in running a biaxial strain test): traction and compression in longitudinal direction 1, traction and compression in transversal direction 2 and pure shear. The ultimate values of deformation measured by the gauges at failure are reported in Table 2.

The numerical model previously defined is implemented using a macro routine compiled in the APDL language in the commercial FE code ANSYS $^{\circledR}$. The characterization tests are simulated in order to find the optimal value for $\alpha$. The model is meshed using linear 3D brick elements and the undamaged elastic properties of the material are taken from Table 1 . The slightly unsymmetric

Table 1. Elastic properties of the unidirectional laminate (superscript $T$ for tension, $C$ for compression)

\begin{tabular}{|c|c|c|c|}
\hline$E_{11}{ }^{T}=90 \mathrm{GPa}$ & $E_{11}{ }^{C}=83.5 \mathrm{GPa}$ & $E_{22}{ }^{T}=E_{33}{ }^{T}=9 \mathrm{GPa}$ & $E_{22}{ }^{C}=E_{33}{ }^{C}=8.5 \mathrm{GPa}$ \\
\hline$v_{12}=v_{13}=0.23$ & $v_{23}=0.3$ & $G_{12}=G_{13}=2.6 \mathrm{GPa}$ & $G_{23}=34 \mathrm{GPa}$ \\
\hline
\end{tabular}

Table 2. Deformation at failure for the unidirectional laminate (subscript $T$ for tension, $C$ for compression)

\begin{tabular}{|c|c|c|c|c|}
\hline$\varepsilon_{1 u T}=0.9 \%$ & $\varepsilon_{1 u C}=-0.3 \%$ & $\varepsilon_{2 u T}=0.5 \%$ & $\varepsilon_{2 u C}=-0.6 \%$ & $\gamma_{12 u}=2 \%$ \\
\hline
\end{tabular}


behaviour in traction-compression is also accounted for, defining an internal procedure by which the proper modulus is chosen when the overall stress state in one element is in traction or in compression. The analysis is performed with a nonlinear incremental-iterative procedure. The value $\alpha=20$ seems to be the best compromise to have accuracy in results and a reasonable computational time. Results, in terms of stress/strain plots, are shown as example in Figure 4 and 5 for the cases of traction in direction 2 and compression in direction 2. A good agreement with experimental results is achieved also for the other tests.

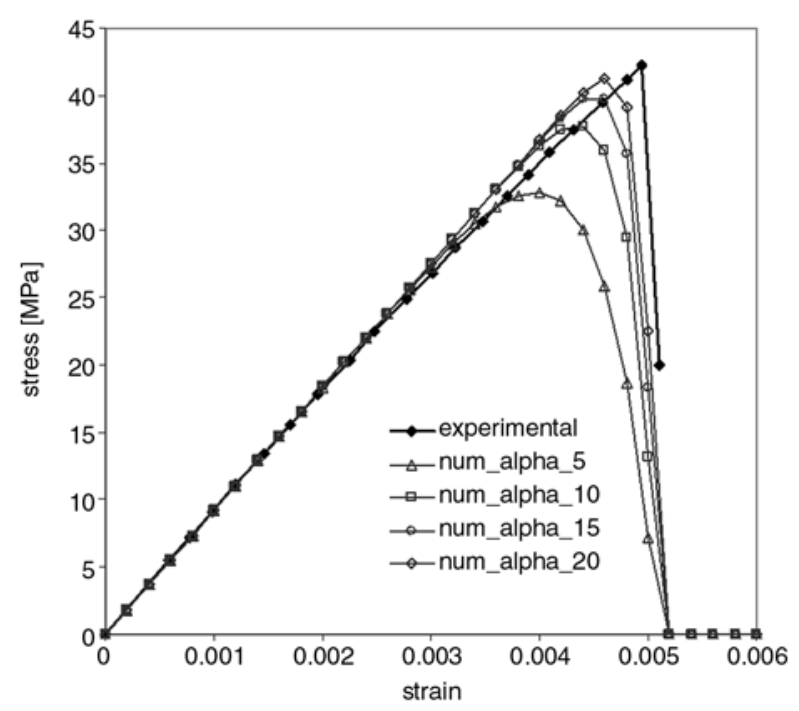

Figure 4. Comparison between experimental results and numerical simulations for tensile test in direction 2

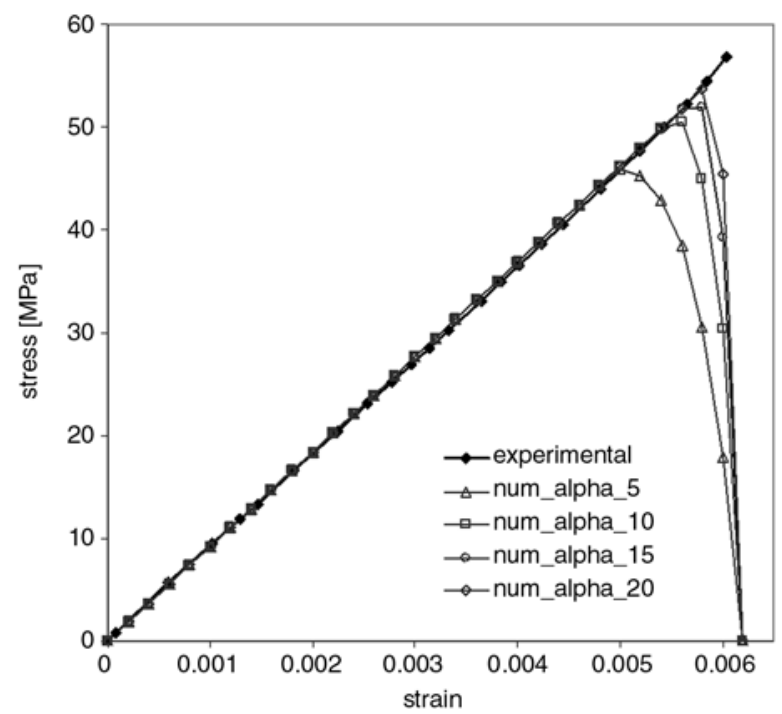

Figure 5. Comparison between experimental results and numerical simulations for compressive test in direction 2

\subsection{Buckling tests}

The buckling behaviour of thin composite specimens is studied with the aim to numerically reproduce the phenomenon during the entire process, that is pre-buckling, post-buckling and failure. Experimental tests are performed in laboratory with a DC servo-motor testing machine. Two different lay-ups are studied: unidirectional $[0]_{12}$ and crossply $[0 / 90]_{3 s}$. The dimensions of the specimens for unidirectional specimens are: gage length $L=120 \mathrm{~mm}$ ( $80 \mathrm{~mm}$ must be added because of the portion required by the grips), width $B=15 \mathrm{~mm}$, thickness $H=2.65 \mathrm{~mm}$ (Figure 6). For cross-ply specimens the dimensions are: gage length $L=87 \mathrm{~mm}$ ( $80 \mathrm{~mm}$ must be added because of the portion required by the grips), width $B=15 \mathrm{~mm}$, thickness $H=2.30 \mathrm{~mm}$. The gage length of the cross-ply specimens has been reduced with respect to unidirectional specimens because the lower value of $E_{x}$ and of the thickness would lead to very small buckling loads for the cross-ply specimens and the results would be more influenced by possible errors in the experimental setup.

The specimens are fixed at the ends (Figure 7) and the tests are run under displacement control with a constant cross-head speed of $2 \mathrm{~mm} / \mathrm{min}$. During the tests the applied axial displacement, the out of plane displacement of the mid-span section and the reaction force of the specimen are recorded.

Five specimens are tested for the unidirectional composite. Each of the diagrams show the typical aspect of the load/displacement plot during a buckling test (Figure 8). During pre-buckling (up to an average applied displacements equal to $0.2 \mathrm{~mm}$ )

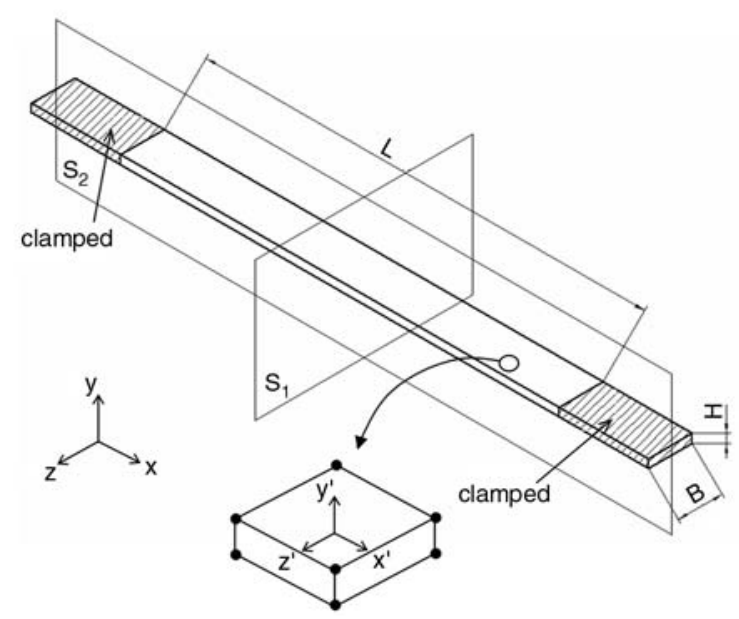

Figure 6. Analysed model ( $\mathrm{S} 1$ and $\mathrm{S} 2$ are planes of symmetry) and a magnified SOLID45 


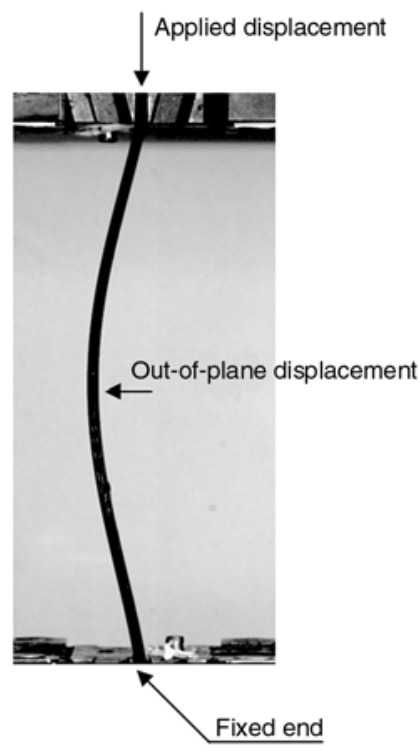

Figure 7. Specimen tested under buckling

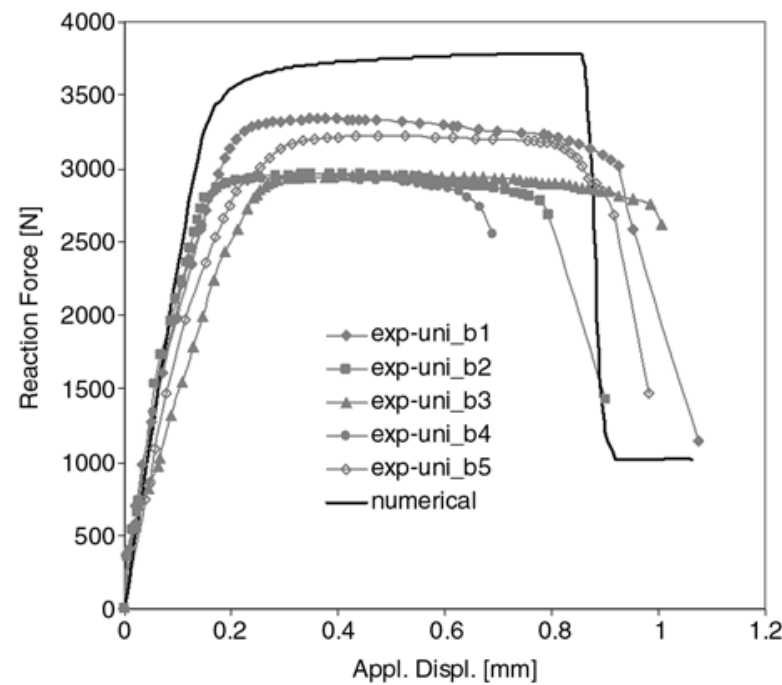

Figure 8. Load/applied displacement diagram for unidirectional specimens: experimental and numerical results

the reaction force increases pseudo-linearly with the applied displacement. Then, at the moment of buckling, the reaction force reaches a plateau and a macroscopic out-of-plane displacement becomes visible. At the end, the reaction force abruptly drops down when failure occurs in the specimens. A significant scattering in the displacements corresponding to the failure of the specimen is found with values that change from $0.7 \mathrm{~mm}$ to $1 \mathrm{~mm}$. The scattering in the buckling load is within the $10 \%$ of the average value. Breakage of the material is observed in correspondence of the mid-span section on the side where compression is present. The crack grows very rapidly through the thickness from the side in compression to the side in traction.

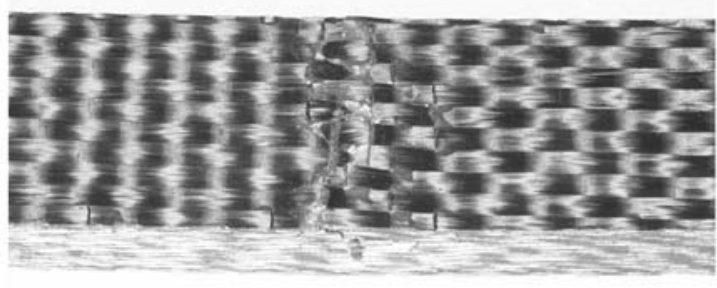

Figure 9. The damaged surface in compression during buckling of a unidirectional specimen

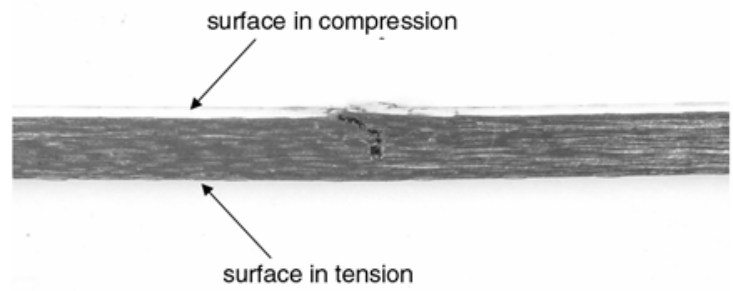

Figure 10. Particular of the through-the-thickness crack of the damaged unidirectional specimen

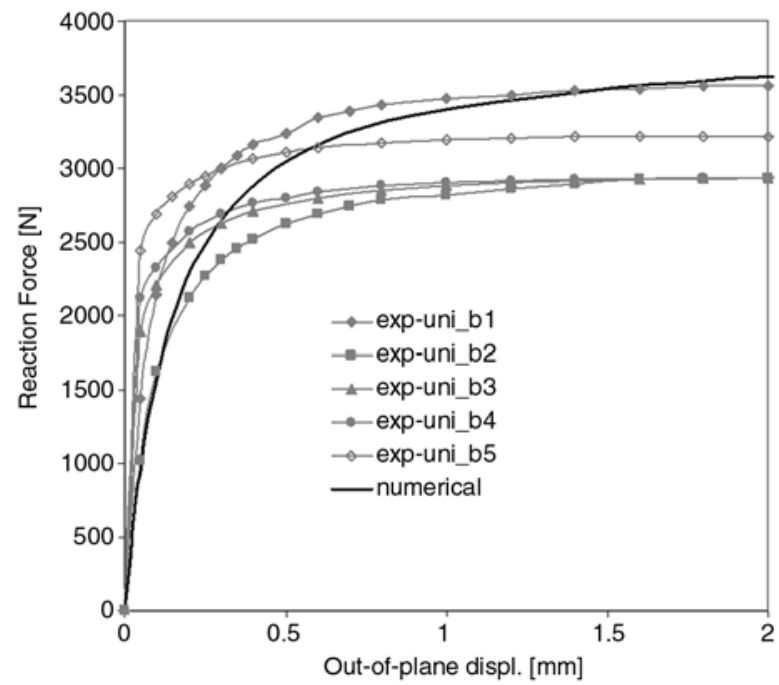

Figure 11. Load/out-of-plane displacement diagram for unidirectional specimens: experimental and numerical results

Figure 9 shows the cracked region of one specimen (side in compression during buckling): there's no evidence of transverse tensile failure due to the Poisson ratio effect and neither delamination can be observed; a few fibre breakages appear on the surface; the main loss of load-carrying capability is due to the through-the-thickness failure of fibres and matrix, as the close-up in Figure 10 illustrates. Figure 11 shows the reaction force as function of the out of plane displacement of the mid-span section. It is evident that, before buckling occurs, very small displacements can be observed, but, at the moment of buckling, the shape of the specimen changes abruptly and the out-of-plane displacement grows up to the final failure. 


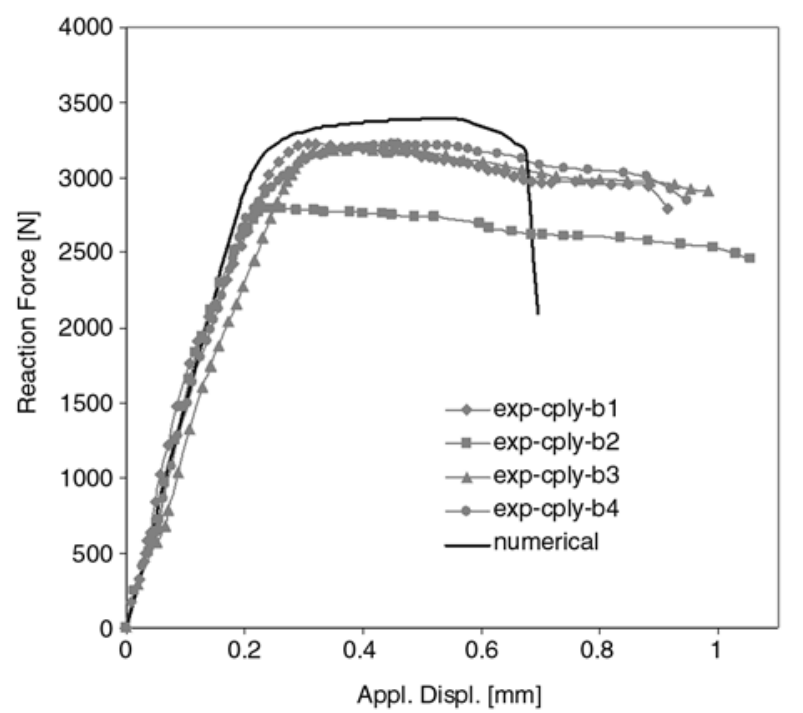

Figure 12. Load/applied displacement diagram for crossply specimens: experimental and numerical results

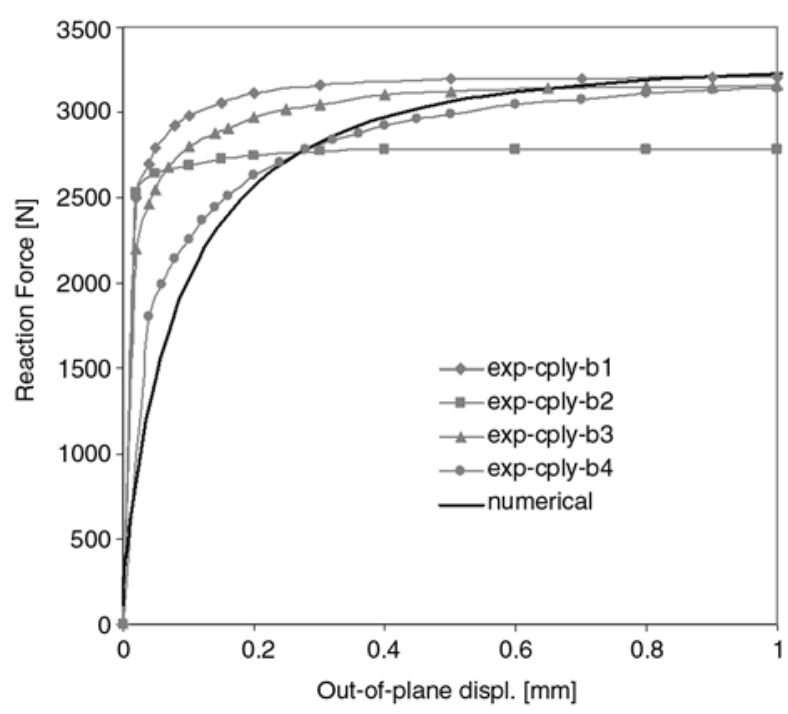

Figure 13. Load/out-of-plane displacement diagram for cross-ply specimens: experimental and numerical results

Similar behaviour is observed for cross-ply specimens. Four specimens are tested and very similar plots are obtained except for the specimen cply_b2 (Figure 12). Differently from unidirectional specimens, failure can be observed both in the mid-span section and in proximity of the grips. The out-ofplane displacements are shown in Figure 13.

\subsection{Numerical simulation of buckling tests}

The computational effort in FEM simulations can be lowered considering the symmetry of the problem with respect to plane $\mathrm{S} 1$ and plane $\mathrm{S} 2$ (Figure 6). For both the lay-ups a quarter of the entire model can be modelled. The elements adopted for the mesh are the linear 8-noded bricks SOLID45 and the material is defined as orthotropic whose elastic characteristics are summarized in Table 1. The axis 1 of the composite lamina is aligned with the $x$ ' axis of the local coordinate system of the element. Each ply of the laminate is meshed with a layer of bricks with the $x$ ' axis aligned to the global $x$ or $z$ axis depending if the lamina is oriented at $0^{\circ}$ or $90^{\circ}$ respectively. The dimensions of the elements are the result of previous convergence analyses [8]. For an accurate reproduction of experiments, also the clamped region of the specimen is modelled.

In such a situation of symmetry, small unbalance forces are needed to favour the instability. These forces are applied in the mid-span section of the specimens in the y direction and their entity can be quantified around $1 / 1000$ of the critical loads.

Load/displacement responses obtained in the tests are compared with results of FEM analyses in Figure 8 for unidirectional specimens. A good agreement can be observed between the two procedures. The difference between FEM result and the average result from experiments can be calculated as the $16 \%$ of the critical load $(P=3700 \mathrm{~N}$ from FEM analyses, $P=3100$ from experiments), and the displacement at failure from FEM is within the interval $0.7-1 \mathrm{~mm}$ observed from tests.

The simple Euler's theory leads to the calculation of the buckling load of a slender beam as follows:

$P_{E u}=\frac{4 \pi^{2} E_{11} I}{L^{2}}$

where $I$ is the moment of inertia, is $P=5870 \mathrm{~N}$, definitely higher than the one carried out from tests. We must recall that analytical theory by Euler assumes some simplifications (ideal clamping at the ends, undamaged and isotropic material, validity of the elastic-beam theory) that can cause nonnegligible overestimations of the critical load. Furthermore, the finite width effect must be accounted for, as the 3D FEM simulations confirms. In fact if we enlarge the map of the Tsai-Wu index $\Psi$ in proximity of the mid-section (Figure 14) when failure takes place, we could notice a gradient of this index from the plane of symmetry $\mathrm{S} 2$ to the free edge. Failure starts to grow from the side in compression to the side in traction, as observed from experiments. In Figure 15 for the 


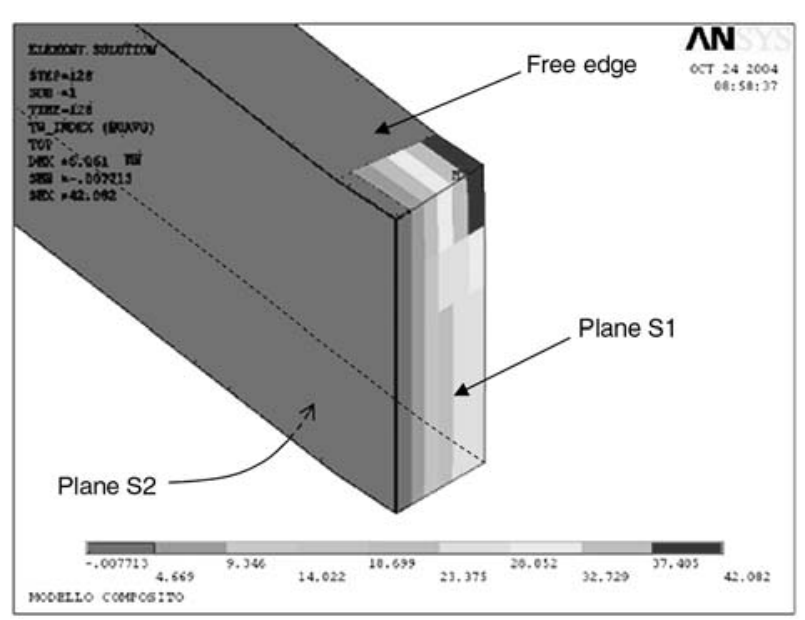

Figure 14. Map of the Tsai-Wu index at failure for a unidirectional specimen: particular in proximity of the mid-span section

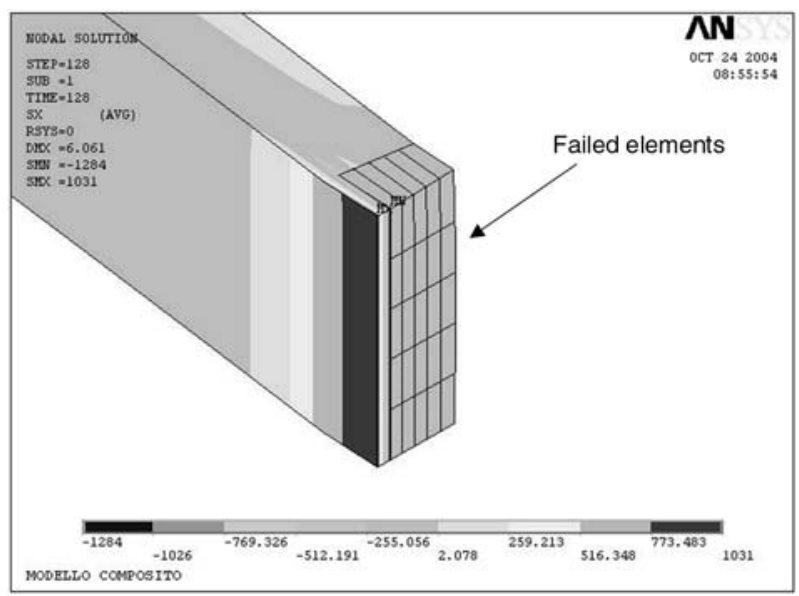

Figure 15. Map of the $\sigma_{x}$ stress at failure for a unidirectional specimen: particular in proximity of the mid-span section

same region the map of $\sigma_{x}$ stress is reported; all the highlighted elements are completely failed elements $(D>1)$ and their elastic modulus is zero. Figure 13 shows comparison between experiments and simulations for the out-of-plane displacement of the mid-span section. Results are in very good agreement and the numerical plot lies between the experimental ones.

Figure 12 illustrates the load/displacement response of tests on cross-ply laminates compared with results from FEA. A very good correspondence can be observed between the load predicted by the simulations and the load from tests $(P=3300 \mathrm{~N}$ from simulations, an average value $P=3200 \mathrm{~N}$ from tests). Some differences, on the contrary, can be seen in the displacement at failure, underestimated by FEM. One of the possible reasons for this difference is that the strength parameter related to the

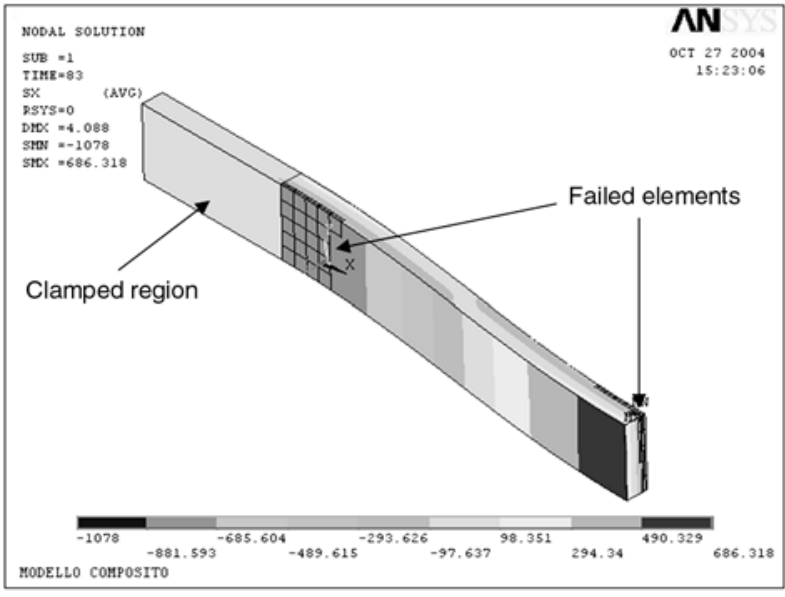

Figure 16. Map of the $\sigma_{x}$ stress at failure for a cross-ply specimen

biaxial strain at failure is evaluated with Eq. (5b) and is not obtained from tests: due to the different elastic characteristics between laminae at $0^{\circ}$ and at $90^{\circ}$, for a cross-ply composite this strength parameter can play a significant role in the failure criterion. It is also remarkable that the zone of failure are similar to the one observed in the tests and are different from those characterizing failure in unidirectional composites. Figure 16 shows the map of the axial $\sigma_{x}$ stress in the specimen: the failed regions are located in the mid-span section and in proximity of the grips. In both regions only $0^{\circ}$ plies show cracked elements on the surface in compression; these cracks propagate in the axial direction (see highlighted elements). The out-of-plane displacement of the mid-span section is reported in Figure 13: results show that FEM displacement grows more slowly than the test results in the first part of the diagram.

\section{Conclusions}

In this paper a continuum damage model for composite materials, based on the Tsai-Wu failure criterion, has been developed and implemented in a commercial FE code. This model is completely defined once the strength parameters of the failure criterion are known: to do that, few characterization tests must be done on the material. Buckling behaviour of thin composite specimens has been studied and numerical results have been compared with those carried out from experimental tests. We can summarize the results as follows.

- The proposed numerical model is general and can be adopted to simulate different phenomena 
involving a progressive damaging of composite materials.

- The power-law adopted to link the damage $D$ to the Tsai-Wu index $\Psi$ well suits to simulate a fastdamaging process with small non-linearities (as usual in CFRP), when conveniently high values are used for the exponent $\alpha$.

- The damage model is isotropic (one value for the exponent $\alpha$ is enough to follow degradation of the composite material in its principal directions) and irreversible (the damage $D$ grows monotonically from 0 to 1 ).

- Experiments of buckling of clamped-clamped composite specimens revealed a great inaccuracy of the Euler's theory for the prediction of the critical load. The overestimation of the load obtained with this approach can cause a wrong design of a structure.

- The good agreement between experimental results and FE results, both for unidirectional and cross-ply laminates, gives reliability to this numerical procedure and affords an accurate prediction of the behaviour of composites when dealing with compression-driven instability.

- Analyses and experiments have been performed on unidirectional and cross-ply laminates. Angleply configurations could also be studied with no modification of the formulation. Other materials should be tested in order to understand the general validity of this damage model and the relation between the exponent $\alpha$ and the elastic properties of the material.

\section{References}

[1] Kardomateas G. A., Dancila D. S.: Buckling of moderately thick orthotropic columns: comparison of an elastic solution with the Euler and Engesser/Haringx/ Timoshenko formulae. International Journal of Solids and Structures, 34, 341-357 (1997).

[2] Chen H. P.: Shear deformation theory for compressive delamination buckling and growth. AIAA Journal, 29, 813-819 (1991).

[3] Shu D.: Buckling of multiple delaminated beams. International Journal of Solids and Structures, 35, 1451-1465 (1998).

[4] Kyoung W. M., Kim C. G.: Delamination buckling and growth of composite laminated plates with trans- verse shear deformation. Journal of Composite Materials, 29, 2047-2068 (1995).

[5] Kyoung W. M., Kim C. G., Hong C. S.: Buckling and postbuckling behavior of composite cross-ply laminates with multiple delaminations. Composite Structures, 43, 257-274 (1998).

[6] Hwang S. F., Liu G. H.: Buckling behavior of composite laminates with multiple delaminations under uniaxial compression. Composite Structures, 53, 235243 (2001).

[7] Hwang S. F., Mao C. P.: Failure of delaminated interply hybrid composite plates under compression. Composites Science and Technology, 61, 1513-1527 (2001).

[8] Cappello F., Tumino D.: Numerical analysis of composite plates with multiple delaminations subjected to uniaxial buckling load. Composites Science and Technology, 66, 264-272 (2006).

[9] Agarwal B. D., Broutman L. J.: Analysis and performance of fiber composites. John Wiley \& Sons, New York (1980)

[10] Swanson S. R.: Introduction to design and analysis with advanced composite materials. Prentice Hall, Upper Saddle River (1997).

[11] Lemaitre J., Chaboche J. L.: Mechanics of solid materials. Cambridge University Press, Cambridge (2000).

[12] de Borst R.: Fracture in quasi-brittle materials: a review of continuum damage-based approaches. Engineering Fracture Mechanics, 69, 95-112 (2002).

[13] Simo J. C., Ju J. W.: Strain and stress-based continuum damage models, Part I: Formulation. International Journal of Solids and Structures, 23, 821-840 (1987).

[14] Kwon Y. W., Liu C. T.: Study of damage evolution in composites using damage mechanics and micromechanics. Composite Structures, 38, 133-139 (1997).

[15] Peerlings R. H. J., Brekelmans W. A. M., de Borst R., Geers M. G. D.: Gradient-enhanced damage modelling of high-cycle fatigue. International Journal for Numerical Methods in Engineering, 49, 1547-1569 (2000).

[16] Raghavan P., Ghosh S.: A continuum damage mechanics model for unidirectional composites undergoing interfacial debonding. Mechanics of Materials, 37, 955-979 (2005).

[17] ASTM D3039/D3039M-93. Standard test method for tensile properties of polymer matrix composite materials (1993).

[18] ASTM D3410-87. Standard test method for compressive properties of unidirectional or crossply fiber-resin composites (1987).

[19] ASTM D3846. Test method for in-plane shear strength of reinforced plastics (1994). 